\section{Hier steht eine Anzeige.}

Springer

Verschluss eines Vorhofseptumdefekts

\title{
Plättchenhemmer schützen vor Neuauftreten von Migräne
}

Fragestellung: Kann eine Behandlung mit Thrombozytenfunktionshemmern das Neuauftreten einer Migräne nach Verschluss eines kardialen Vorhofseptumdefekts (ASD) das Auftreten einer Migräne verhindern?

Hintergrund: Vorhofseptumdefekte werden heute interventionell durch Verschlusssysteme behandelt. Bei $15 \%$ aller Patienten tritt nach dem ASD-Verschluss eine Migräne auf. Die Autoren wollten untersuchen, ob eine Behandlung mit Thrombozytenfunktionshemmern das Neuauftreten einer Migräne nach ASD-Verschluss verhindern kann.

Patienten und Methodik: Es handelt sich um eine randomisierte doppelblinde Studie an sechs Universitätskliniken in Kanada. Von Dezember 2008 bis Februar 2015 wurden 171 Patienten eingeschlossen, bei denen ein Vorhofseptumdefekt verschlossen wurde und die bis dahin keine Migräne hatten. Die Patienten erhielten entweder über einen Zeitraum von drei Monaten eine duale Plättchenhemmung mit Acetylsalicylsäure (ASS) plus Clopidogrel oder eine ASS-Monotherapie plus Placebo. Primärer Studienendpunkt waren Migränetage innerhalb der ersten drei Monate nach ASD-Verschluss.

Ergebnisse: Die Patienten waren im Mittel 49 Jahre alt und $62 \%$ waren Frauen. In die endgültige Analyse gingen 84 Patienten in der Kombinationstherapiegruppe und 87 in der Monotherapiegruppe ein. Die mittlere Zahl der Migränetage pro Monat betrug 0,4 in der Gruppe der Patienten, die ASS und Clopidogrel erhielten und 1,4 in der ASSMonotherapiegruppe. Dieser Unterschied war statistisch signifikant. Die Zahl der Migränetage in drei Monaten betrug einen Tag in der Kombinationstherapiegruppe und 3,8 Tage in der Monotherapiegruppe. Die Rate neu aufgetretener Migräne betrug 9,5\% in der Kombinationstherapiegruppe $(\mathrm{n}=8)$ und $21,8 \%$ in der Monotherapiegruppe $(n=19)$. Bei den

Rodés-Cabau J, Horlick E, Ibrahim R et al. Effect of clopidogrel and aspirin vs aspirin alone on migraine headaches after transcatheter atrial septal defect closure: The CANOA Randomized Clinical Trial. JAMA 2015; 314: 2147-54 meisten Patienten handelte es sich um eine Migräne mit Aura.

Schlussfolgerungen: Bei Patienten, bei denen ein interventioneller ASD-Verschluss durchgeführt wird, tritt bei einer Kombinationstherapie von Clopidogrel und ASS seltener eine Migräne auf, als bei Patienten, die allein mit ASS behandelt werden.

\section{- Kommentar von Hans-Christoph Diener, Essen}

\section{Kombinationstherapie deutlich wirksamer}

Es ist eine interessante Beobachtung, dass bei Verschlüssen von Vorhofseptumdefekten bei bis zu 20\% der Betroffenen, überwiegend Frauen, eine Migräne auftreten kann. Der Pathomechanismus dieses Phänomens ist bisher nicht verstanden. Bei allen Verschlusssystemen wird über einen Zeitraum von drei Monaten ein Thrombozytenfunktionshemmer gegeben, bis das device endothelialisiert ist. Diese Studie zeigt, dass eine Kombinationstherapie von Acetylsalicylsäure und Clopidogrel wirksamer als eine Monotherapie mit Acetylsalicylsäure ist, um das Neuauftreten einer Migräne zu verhindern. $\mathrm{Ob}$ dies auch für die normale Migräneprophylaxe gilt, ist allerdings bisher nicht bekannt. Es gibt nur zwei kleinere Studien die zeigten, dass Acetylsalicylsäure eine moderate migräneprophylaktische Wirkung hat. 